Chirurg $2021 \cdot 92: 846$

https://doi.org/10.1007/s00104-021-01477-8

Angenommen: 6. Juli 2021

Online publiziert: 16. August 2021

(c) Springer Medizin Verlag $\mathrm{GmbH}$, ein Teil von Springer Nature 2021

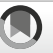

\section{Ist die CME bei der laparoskopi- schen Hemicolektomie rechts sicher durchführbar?}

\author{
S. Axt $\cdot$ M. Anthuber ${ }^{1}$ \\ ${ }^{1}$ Klinik für Allgemein-, Viszeral- und Transplantationschirurgie, Universitätsklinikum Augsburg, Augsburg, \\ Deutschland
}

Originalpublikation

Di Buono G, Buscemi S, Agrusa A et al (2021) Feasibility and safety of laparoscopic complete mesocolic excision (CME) for right-sided colon cancer. Ann Surg 274:57-62

Hintergrund. Die Technik der totalen mesorektalen Exzision ist aufgrund des deutlich besseren onkologischen Outcomes zum Goldstandard in der Behandlung des Rektumkarzinoms erklärt worden. Analog hierzu wurde von Hohenberger 2009 das Konzept der CME („,complete mesocolic excision“) mit zentraler Ligatur der Gefäße (CVL) bei der Hemikolektomie rechts implementiert [2]. Mit zunehmender Anzahl an laparoskopischen Rechtshemikolektomien kam es jedoch zu einem deutlich erhöhten Rezidivrisiko, vermutlich durch eine inadäquate Lymphknotendissektion. Vor diesem Hintergrund untersucht die vorliegende Studie, ob auch laparoskopisch eine adäquate Lymphknotendissektion beim rechtsseitigen Kolonkarzinom im Sinne einer CME möglich und auch sicher durchführbar ist.

Methoden. Die eingeschlossenen Patienten erhielten eine laparoskopische Hemikolektomie rechts und wurden entweder in die Gruppe der konventionellen Operationstechnik (NCME[Non complete

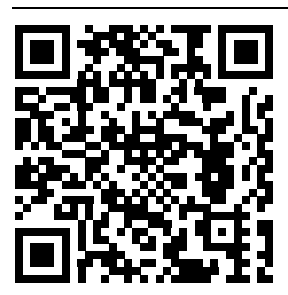

QR-Code scannen \& Beitrag online lesen mesoccolic excision]-Gruppe) oder in die CME-Gruppe randomisiert. Das Patientenkollektiv wurde innerhalb eines Zeitraums von 30 Tagen hinsichtlich primärer Endpunkte wie Operationszeit, Blutverlust, Komplikationen, Konversions- und Anastomoseninsuffizienzrate evaluiert. Sekundäre Endpunkte waren sämtliche postoperativen Komplikationen. Zudem erfolgte die Auswertung der histologischen Ergebnisse hinsichtlich Länge des Präparates sowie Anzahl der gewonnenen Lymphknoten als Marker für die Qualität der CME.

Ergebnisse. Ausgewertet wurden insgesamt 132 Patienten. In der CME-Gruppe war die Operationszeit signifikant länger (216 vs. $191 \mathrm{~min}$ ), zudem zeigten sich ein durchschnittlich längeres Präparat (34,3 vs. $29,3 \mathrm{~cm}$ ) sowie eine höhere Anzahl an resezierten Lymphknoten (23,8 vs. 16,6). Hinsichtlich der weiteren Endpunkte konnte zwischen beiden Gruppen kein signifikanter Unterschied festgestellt werden.

\section{Kommentar}

Die deutlich höhere Lymphknotenausbeute in der CME-Gruppe legt die Vermutung nahe, dass bei nicht durchgeführter CME das postoperative $\mathrm{N}$-Stadium oft fälschlicherweise als zu niedrig angegeben wird. Als Erklärung für die signifikant höhere Lymphknotenzahlen vermuten die Autoren die sorgfältige Präparation entlang embryonaler Landmarken sowie der zentralen Ligatur der Gefäße.

Hinsichtlich der Komplikationsrate konnte kein Unterschied in beiden Gruppen festgestellt worden. Dies unterstützt die These von Garcia-Granero et al. [1], dass die schichtgerechte Präparation mit einem niedrigeren Risiko für Blutungen und intraoperative Komplikationen einhergeht.

Als Fazit der vorliegenden Studie kann festgehalten werden kann, dass die laparoskopische CME, sofern sie von gut geschulten Chirurgen durchgeführt wird, machbar und sicher ist und mit einer im Vergleich zur Kontrollgruppe erhöhten Lymphknotenausbeute einhergeht. Das längerfristige Outcome gilt es in weiteren Studien zu evaluieren. Kritisch anzumerken und verwunderlich ist, dass nach den Arbeiten von Hohenberger 2009 und dem Nachweis des Vorteils der CME eine randomisierte Studie zu dieser Fragestellung mit Patientenrekrutierung zwischen 2015 bis 2019 machbar ist.

\section{Korrespondenzadresse}

Prof. Dr. M. Anthuber

Klinik für Allgemein-, Viszeral- und

Transplantationschirurgie, Universitätsklinikum Augsburg

Stenglinstr. 2, 86156 Augsburg, Deutschland matthias.anthuber@uk-augsburg.de

Interessenkonflikt. S. Axt und M. Anthuber geben an, dass kein Interessenkonflikt besteht.

\section{Literatur}

1. Garcia-Granero A, Pellino G, Frasson M et al (2019) The fusion fascia of Fredet: an important embryological landmark for complete mesocolic excision and D3-lymphadenectomy in right colon cancer. Surg Endosc 33:3842-3850

2. Hohenberger W, Weber K, Matzel K et al (2009) Standardized surgery for colonic cancer: complete mesocolic excision and central ligation - technical nots and outcome. Colorectal Dis 11:354-364 